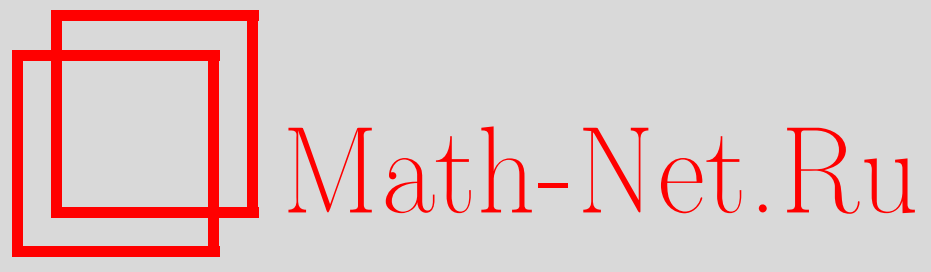

П. А. Крутицкий, Об обратимости одного оператора, УМН, 2014, том 69, выпуск 1, 171-172

DOI: https://doi.org/10.4213/rm9540

Использование Общероссийского математического портала Math-Net.Ru подразумевает, что вы прочитали и согласны с пользовательским соглашением http://www . mathnet.ru/rus/agreement

Параметры загрузки:

IP: 35.173 .137 .237

26 апреля 2023 г., 15:35:41

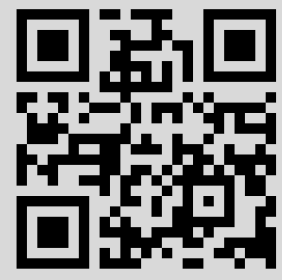




\section{Об обратимости одного оператора}

\section{П. А. Крутицкий}

Для функций $V(x)$, заданных в декартовых координатах $x=\left(x_{1}, x_{2}\right)$ на плоскости $\mathbb{R}^{2}$, введем преобразование Фурье

$$
\widehat{G}(\xi)=\int_{\mathbb{R}^{2}} G(x) \exp (-2 \pi i x \cdot \xi) d x, \quad \xi=\left(\xi_{1}, \xi_{2}\right), \quad x \cdot \xi=x_{1} \xi_{1}+x_{2} \xi_{2} .
$$

Образы Фурье обозначаются крышкой. Там, где преобразование Фурье не существует в обычном смысле, оно понимается в смысле теории обобщенных функций. Используя [1] и вычисляя преобразование Фурье для функции $K(x)=|x|^{-1} \exp (-k|x|)$, где $|x|=\left(x_{1}^{2}+x_{2}^{2}\right)^{1 / 2}$ и $k>0$, получим $\widehat{K}(\xi)=\left(k_{0}^{2}+|\xi|^{2}\right)^{-1 / 2}$, где $|\xi|=\left(\xi_{1}^{2}+\xi_{2}^{2}\right)^{1 / 2}$, $k_{0}=k /(2 \pi)$. Введем шкалу соболевских пространств $H^{s}\left(\mathbb{R}^{2}\right),-\infty<s<\infty$, с нормой

$$
\|G\|_{H^{s}\left(\mathbb{R}^{2}\right)}^{2}=\int_{\mathbb{R}^{2}}\left(1+|\xi|^{2}\right)^{s}|\widehat{G}(\xi)|^{2} d \xi .
$$

Пусть $K(x-y)=\left(\left(x_{1}-y_{1}\right)^{2}+\left(x_{2}-y_{2}\right)^{2}\right)^{-1 / 2} \exp \left(-k\left(\left(x_{1}-y_{1}\right)^{2}+\left(x_{2}-y_{2}\right)^{2}\right)^{1 / 2}\right)$. Рассмотрим оператор свертки $A$, действующий на функции, заданные в $\mathbb{R}^{2}$ :

$$
(A G)(x)=\int_{\mathbb{R}^{2}} G(y) K(x-y) d y .
$$

По теореме о свертке [2]: $(\widehat{A G})(\xi)=\widehat{K}(\xi) \widehat{G}(\xi)$. Покажем, что оператор $A$ действует из $H^{-1 / 2}\left(\mathbb{R}^{2}\right)$ в $H^{1 / 2}\left(\mathbb{R}^{2}\right)$ и при этом ограничен:

$$
\begin{aligned}
\|A G\|_{H^{1 / 2}\left(\mathbb{R}^{2}\right)}^{2} & =\int_{\mathbb{R}^{2}}\left(1+|\xi|^{2}\right)^{1 / 2}|\widehat{A G}(\xi)|^{2} d \xi=\int_{\mathbb{R}^{2}}\left(1+|\xi|^{2}\right)^{1 / 2}|\widehat{K}(\xi)|^{2}|\widehat{G}(\xi)|^{2} d \xi \\
& =\int_{\mathbb{R}^{2}} \frac{\left(1+|\xi|^{2}\right)^{1 / 2}}{k_{0}^{2}+|\xi|^{2}}|\widehat{G}(\xi)|^{2} d \xi=\int_{\mathbb{R}^{2}} \frac{1+|\xi|^{2}}{k_{0}^{2}+|\xi|^{2}}\left(1+|\xi|^{2}\right)^{-1 / 2}|\widehat{G}(\xi)|^{2} d \xi \\
& \leqslant \max \left\{k_{0}^{-2}, 1\right\} \int_{\mathbb{R}^{2}}\left(1+|\xi|^{2}\right)^{-1 / 2}|\widehat{G}(\xi)|^{2} d \xi=\mathrm{const}\|G\|_{H^{-1 / 2}\left(\mathbb{R}^{2}\right)}^{2}
\end{aligned}
$$

Здесь использована оценка $\left(1+|\xi|^{2}\right)\left(k_{0}^{2}+|\xi|^{2}\right)^{-1} \leqslant \max \left\{k_{0}^{-2}, 1\right\}$. Пространства $H^{-1 / 2}\left(\mathbb{R}^{2}\right)$ и $H^{1 / 2}\left(\mathbb{R}^{2}\right)$ являются двойственными в смысле скалярного произведения в $L_{2}\left(\mathbb{R}^{2}\right)$.

$\mathrm{B}$ плоскости $\mathbb{R}^{2}$ рассмотрим конечное число замкнутых компактных липшицевых областей. Будем предполагать, что эти области не имеют общих точек друг с другом, в частности не имеют общих граничных точек. Совокупность этих областей обозначим $\Omega$. Тем самым, $\Omega$ - (несвязная) замкнутая липшицева область в $\mathbb{R}^{2}$. Введем соболевские пространства на $\Omega$ :

$$
\begin{aligned}
\widetilde{H}^{-1 / 2}(\Omega) & =\left\{u: u \in H^{-1 / 2}\left(\mathbb{R}^{2}\right), \operatorname{supp} u \subset \Omega\right\}, \\
H^{1 / 2}(\Omega) & =\left\{u: u=\left.U\right|_{\Omega} \text { для некоторого } U \in H^{1 / 2}\left(\mathbb{R}^{2}\right)\right\}, \\
\|u\|_{\widetilde{H}^{-1 / 2}(\Omega)} & =\|u\|_{H^{-1 / 2}\left(\mathbb{R}^{2}\right)}, \quad\|u\|_{H^{1 / 2}(\Omega)}=\min _{\left.U\right|_{\Omega}=u, U \in H^{1 / 2}\left(\mathbb{R}^{2}\right)}\|U\|_{H^{1 / 2}\left(\mathbb{R}^{2}\right)} .
\end{aligned}
$$

Можно показать [2; теорема 3.30], что если $\Omega$ - липшицева область, то пространства $\widetilde{H}^{-1 / 2}(\Omega)$ и $H^{1 / 2}(\Omega)$ являются двойственными в смысле скалярного произведения в $L_{2}(\Omega)$, т. е. если $\mu \in \widetilde{H}^{-1 / 2}(\Omega)$ и $g \in H^{1 / 2}(\Omega)$, то определено скалярное произведение

$$
\begin{aligned}
(\mu, g)_{L_{2}(\Omega)} & =\int_{\Omega} \overline{\mu(x)} g(x) d x=\int_{\mathbb{R}^{2}} \overline{\mu(x)} G(x) d x=(\mu, G)_{L_{2}\left(\mathbb{R}^{2}\right)} \\
& =(\hat{\mu}, \widehat{G})_{L_{2}\left(\mathbb{R}^{2}\right)}=\int_{\mathbb{R}^{2}} \overline{\hat{\mu}(\xi)} \widehat{G}(\xi) d \xi,
\end{aligned}
$$

DOI: $10.4213 / \mathrm{rm} 9540$ 
где функция $G \in H^{1 / 2}\left(\mathbb{R}^{2}\right)$ такова, что $g=\left.G\right|_{\Omega}$ (по определению пространства $H^{1 / 2}(\Omega)$ функция $G$ всегда существует). Четвертое равенство в последней формуле гарантируется теоремой Планшереля [2; теорема 3.12$]$.

На функциях $\mu$, определенных в области $\Omega$, рассмотрим оператор $A_{\Omega}$

$$
\left(A_{\Omega} \mu\right)(x)=\int_{\Omega} \mu(y) K(x-y) d y, \quad x \in \Omega,
$$

который задает некоторую функцию на $\Omega$. Пусть $\mu \in \widetilde{H}^{-1 / 2}(\Omega) \subset H^{-1 / 2}\left(\mathbb{R}^{2}\right)$, тогда

$$
\left(A_{\Omega} \mu\right)(x)=\int_{\Omega} \mu(y) K(x-y) d y=\left.\left(\int_{\mathbb{R}^{2}} \mu(y) K(x-y) d y\right)\right|_{x \in \Omega}=\left.(A \mu)(x)\right|_{x \in \Omega} .
$$

Поскольку оператор $A$ действует из $H^{-1 / 2}\left(\mathbb{R}^{2}\right)$ в $H^{1 / 2}\left(\mathbb{R}^{2}\right)$, то оператор $A_{\Omega}$ действует из $\widetilde{H}^{-1 / 2}(\Omega)$ в $H^{1 / 2}(\Omega)$ в силу определения пространства $H^{1 / 2}(\Omega)$.

Выше было показано, что оператор $A$ ограничен при действии из $H^{-1 / 2}\left(\mathbb{R}^{2}\right)$ в $H^{1 / 2}\left(\mathbb{R}^{2}\right)$, поэтому и при действии из $\widetilde{H}^{-1 / 2}(\Omega) \subset H^{-1 / 2}\left(\mathbb{R}^{2}\right)$ в $H^{1 / 2}\left(\mathbb{R}^{2}\right)$ он также ограничен. Если множество функций ограничено по норме в $H^{1 / 2}\left(\mathbb{R}^{2}\right)$ константой, то и множество сужений этих функций на $\Omega$ будет ограничено по норме в $H^{1 / 2}(\Omega)$, причем той же константой в силу определения нормы в $H^{1 / 2}(\Omega)$. Следовательно, оператор $A_{\Omega}$ ограничен при действии из $\widetilde{H}^{-1 / 2}(\Omega)$ в $H^{1 / 2}(\Omega)$.

Заметим, что справедлива оценка $\left(1+|\xi|^{2}\right)^{1 / 2}\left(k_{0}^{2}+|\xi|^{2}\right)^{-1 / 2} \geqslant\left(\min \left\{k_{0}^{-2}, 1\right\}\right)^{1 / 2}$. Пусть $\mu \in \widetilde{H}^{-1 / 2}(\Omega)$. Учитывая, что пространства $\widetilde{H}^{-1 / 2}(\Omega)$ и $H^{1 / 2}(\Omega)$ - двойственные в смысле скалярного произведения в $L_{2}(\Omega)$, получим

$$
\begin{gathered}
\left(A_{\Omega} \mu, \mu\right)_{L_{2}(\Omega)}=\left(\left.A \mu\right|_{\Omega}, \mu\right)_{L_{2}(\Omega)}=(A \mu, \mu)_{L_{2}\left(\mathbb{R}^{2}\right)}=(\widehat{A \mu}, \hat{\mu})_{L_{2}\left(\mathbb{R}^{2}\right)}=\int_{\mathbb{R}^{2}} \widehat{K}(\xi)|\hat{\mu}(\xi)|^{2} d \xi \\
\quad=\int_{\mathbb{R}^{2}}\left(k_{0}^{2}+|\xi|^{2}\right)^{-1 / 2}|\hat{\mu}(\xi)|^{2} d \xi=\int_{\mathbb{R}^{2}}\left(1+|\xi|^{2}\right)^{-1 / 2}\left(\frac{1+|\xi|^{2}}{k_{0}^{2}+|\xi|^{2}}\right)^{1 / 2}|\hat{\mu}(\xi)|^{2} d \xi \\
\geqslant\left(\min \left\{k_{0}^{-2}, 1\right\}\right)^{1 / 2} \int_{\mathbb{R}^{2}}\left(1+|\xi|^{2}\right)^{-1 / 2}|\hat{\mu}(\xi)|^{2} d \xi=c\|\mu\|_{H^{-1 / 2}\left(\mathbb{R}^{2}\right)}^{2}=c\|\mu\|_{\widetilde{H}^{-1 / 2}(\Omega)}^{2} .
\end{gathered}
$$

Следовательно, оператор $A_{\Omega}$ положителен и ограничен снизу при действии из $\widetilde{H}^{-1 / 2}(\Omega)$ в $H^{1 / 2}(\Omega)$. Поскольку, кроме того, оператор $A_{\Omega}$ ограничен при действии из $\widetilde{H}^{-1 / 2}(\Omega)$ в $H^{1 / 2}(\Omega)$ и пространства $\widetilde{H}^{-1 / 2}(\Omega)$ и $H^{1 / 2}(\Omega)$ - двойственные, то по лемме 2.32 в [2] для оператора $A_{\Omega}$ существует ограниченный обратный оператор. Из приведенных рассуждений вытекает следующая теорема.

Теорема. Для оператора $A_{\Omega}$, действующего из $\widetilde{H}^{-1 / 2}(\Omega)$ в $H^{1 / 2}(\Omega)$, имеется ограниченный обратный оператор $A_{\Omega}^{-1}$.

СледствиЕ. Для любой функции $g \in H^{1 / 2}(\Omega)$ уравнение $A_{\Omega} \mu=g$ имеет в пространстве $\widetilde{H}^{-1 / 2}(\Omega)$ единственное решение.

\section{Список литературы}

[1] Г. Бейтмен, А. Эрдейи, Таблицы интегральных преобразований. Т. 1: Преобразование Фуръе, Лапласа, Меллина, Наука, М., 1969, 343 с. [2] W. McLean, Strongly elliptic systems and boundary integral equations, Cambridge Univ. Press, Cambridge, 2000, xiv +357 pp.

Павел Александрович Крутицкий

(Pavel A. Krutitskii)

Институт прикладной математики

им. М. В. Келдыша РАН

E-mail: biem@mail.ru
Представлено М. И. Зеликиным Принято редколлегией 19.07.2013 